\title{
Compact Stellar Model with Two Different Equations of State
}

\author{
Daddy Balondo Iyela ${ }^{1}$, Nestor Anzola Kibamba ${ }^{2}$ \\ ${ }^{1}$ Department of Physics, Faculty of Science, University of Kinshasa, Kinshasa, Democratic Republic of Congo \\ ${ }^{2}$ Department of Mathematics and Computer Science, Faculty of Science, University of Kinshasa, Kinshasa, Democratic Republic of Congo
}

Email address:

Balondo36@gmail.com (D. B. Iyela), nestoranzola@gmail.com (N. A. Kibamba)

\section{To cite this article:}

Daddy Balondo Iyela, Nestor Anzola Kibamba. Compact Stellar Model with Two Different Equations of State. American Journal of

Electromagnetics and Applications. Vol. 8, No. 1, 2020, pp. 12-17. doi: 10.11648/j.ajea.20200801.12

Received: June 5, 2019; Accepted: August 15, 2019; Published: February 25, 2020

\begin{abstract}
We have modelled a neutral non-homogeneous anisotropic stellar compact object with two distinct equations of state in general relativity framework. We have considered the macroscopic features of a general relativistic gravitating compact object. The equation of state is quadratic in the core and linear in the envelope. There is smooth matching between the core, envelope and the vacuum exterior regions. We found the masses, radii and compactness of some compact objects such PSR J1614-2230, PSR J1903+0327, Vela X-1, SMC-X-1, Cen X-3; which are in agreement with previous investigations. The gravitational potentials and the matter variables are well behaved throughout the stellar structure. We present in particular the variation of the radius in the core and the envelope of the star by changing some parameters values. Physical features of the pulsars PSR J1614-2230 are presented in more details. It observed that the radial pressure in the core is higher than the radial pressure in the envelope. The investigation reveals that the model is physically relevant for the study of observed compact stars.
\end{abstract}

Keywords: General Relativity, Compact Star, Equation of State

\section{Introduction}

The study of the gravitational behavior in superdense astronomical objects is an important area of research in astrophysics in a general relativistic setting. It is believed that the high central densities at the core provide an environment for the relativistic nucleons to convert to hyperons or to generate condensates. The modeling of the interior of stars with a quart has been first performed by the studies [1, 2]. The exact constitution for the matter distribution in the core regions remains a question for deeper investigation. In most studies of highly compact bodies the core is surrounded by an envelope or nuclear crust consisting of baryonic matter. An example of such physical situation is provided by the model with concentric layers of different phases, an inner deconfined quark phase and an outer less compact baryonic layer [3]. In a core envelope model for a relativistic stellar configuration the matter distributions of the two regions have different physical features. In our approach the composition of matter is contained in the equations of state for the envelope and the core. We are concerned with the macroscopic behavior of the matter distribution; it is not possible to completely describe the microphysics in this approach.

Several exact core envelope models in general relativity have been found in the past $[4,5]$. Precise details of the behavior of matter in superdense compact objects are not fully understood. Particular core envelope models for massive relativistic spheres in general relativity have been found in the past. The first exact interior solutions with different energy densities for the core and envelope were found [6-8]. Nonterminating exact solutions for isothermal neutron star interiors which are gravitationally bound and stable were presented by [9]. Models with a parabolic energy density distribution at the core were analyzed by the papers $[10,11]$. The core envelope model related to the compact $\mathrm{X}$ ray binary pulsar Her X-1 for a quark-diquark mixture in equilibrium were investigated by [12]. Exact core envelope models with the core layer having either isotropic or anisotropic pressures were found by $[13,14]$. They also specified the space-time geometry to be parabolic, spheroidal or pseudo spheroidal to solve the field equations.

Modeling a highly compact relativistic gravitating object with a core envelope description is desirable in relativistic astrophysics. The investigations of [15] concerning realistic 
stellar models demonstrate that at very high density ranges beyond $10^{14} \mathrm{gcm}^{3}$, the nuclear matter may have anisotropic pressures and its interactions should be treated relativistic ally. [16] included non-homogeneous matter for describing stellar compact object like neutron stars. The non-homogeneous matter with anisotropic pressures is important feature for a superdense star in hydrostatic equilibrium which satisfies all physical constraint for the core and the envelope [5]. Another important source of anisotropy is intense magnetic fields observed in compact objects like neutron stars, white dwarfs and strange quark stars as pointed out by [17]. The matter distribution should satisfy a barotropic equation of state throughout the star. In our stellar model the equation of state is quadratic in the core region; this ensures that the radial pressures are higher close to the center. Stellar models with a quadratic equation of state have been studied by the studies [19, 20]. The equation of state is linear in the envelope; this also ensures that the radial pressure in the envelope is lower than the core. Stellar models with a linear equation of state have been recently investigated by the studies [21-24].

In this work we find a new core envelope model for astrophysical objects by matching two inner regions with different equations of state. The outer region is described by the Schwarzschild metric. We review the Einstein field equations in Sec. 2. In Sec. 3, we present the exact solutions for the core and the envelope. In Sec. 4, the matching conditions between regions are explored. Masses and radii for the astronomical objects are presented in Table 1. And the matter variables are plotted in Sec. 5. In sec. 6, we analyze the physical features of the model in connection with the pulsar PSR J1614-2230 and the results are shown in Table 2 and Table 3. We briefly summaries the results obtained in this work in Sec. 7.

\section{The Model}

For the interior description of the stellar uncharged nonhomogeneous body, the physical relevant energy momentum tensor is given by

$$
T^{\mu \nu}=\operatorname{diag}\left(-\rho, p_{r}, p_{t}, p_{t}\right)
$$

with $\rho, p_{r}$ and $p_{t}$ being the energy density, radial pressure and the tangential pressure respectively. These quantities are the matter variables. It is convenient to introduce the degree of anisotropy $\Delta=p_{t}-p_{r}$ which vanishes for isotropic pressures. In our study we choose a static field for which the interior metric is given as

$$
d s^{2}=-e^{2 v} d t^{2}+e^{2 \lambda} d r^{2}+r^{2}\left(d \theta^{2}+\sin ^{2} \theta d \phi^{2}\right)
$$

with $v=v(r)$ and $\lambda=\lambda(r)$ being the gravitational potentials. The line element (2) is a reasonable approximation for a highly compact gravitating body such as a neutron star.

The Einstein field equations governing the gravitational behavior of an uncharged sphere with non-uniform matter distribution, give the following

$$
\begin{gathered}
\rho=\frac{1}{r^{2}}\left[r\left(1-e^{-2 \lambda}\right)\right]^{\prime}, \\
p_{r}=-\frac{1}{r^{2}}\left(1-e^{-2 \lambda}\right)+\frac{2 v^{\prime}}{r} e^{-2 \lambda}, \\
p_{t}=e^{-2 \lambda}\left(v^{\prime \prime}+v^{\prime 2}+\frac{v^{\prime}}{r}-\frac{\lambda^{\prime}}{r}-v^{\prime} \lambda^{\prime}\right),
\end{gathered}
$$

where primes represent differentiation with respect to $r$. The nonlinear character of Einstein's field equations makes it difficult to solve them analytically. To find a solution, we need to restrict the behavior of the potentials, prescribe the form of the matter variables and choose a specific equation of state.

In many relativistic stellar configurations the inside matter distribution is made up with two regions: an inner core and outer envelope with different pressures. To model a core envelope star or hybrid star we need to separate space-time into three regions. The three regions consist of the core (region $I, 0 \leq r \leq R_{I}$ ), the envelope (region $I I, R_{I} \leq r \leq R_{I I}$ ) and the exterior (region $I I I, R_{I I} \leq r$ ). The line elements for the three regions are given by

$$
\begin{gathered}
\left.d s^{2}\right|_{I}=-e^{2 v_{I}} d t^{2}+e^{2 \lambda_{I}} d r^{2}+r^{2}\left(d \theta^{2}+\sin ^{2} \theta d \phi^{2}\right) \\
\left.d s^{2}\right|_{I I}=-e^{2 v_{I I}} d t^{2}+e^{2 \lambda_{I I}} d r^{2}+r^{2}\left(d \theta^{2}+\sin ^{2} \theta d \phi^{2}\right) \\
\left.d s^{2}\right|_{I I I}=-\left(1-\frac{2 M}{r}\right) d t^{2}+\left(1-\frac{2 M}{r}\right)^{-1} d r^{2}+r^{2}\left(d \theta^{2}+\sin ^{2} \theta d \phi^{2}\right)
\end{gathered}
$$

In the above (8) is the Schwarzschild exterior solution which relates to the region III which is the exterior of the gravitating star. The stellar boundary has to match smoothly to the Schwarzschild region III for consistency which implies vanishing radial pressure; the condition must hold in all general relativistic models for an isolated matter source.

For physical viability the model should comply with the following requirements in all three regions (core, envelope and exterior):

i. The gravitational potentials and matter variables should be well defined at the centre and regular throughout the star,

ii. The energy density $\rho>0$ and the gradient of density $\rho^{\prime}<0$ in the core and the envelope.

iii. The radial pressure $p_{r}>0$, the tangential pressure $p_{t}>0$, the speed of sound $\frac{d p_{r}}{d \rho} \leq 1$ and the gradient of pressure $\frac{d p_{r}}{d r}<0$ in the core and the envelope,

iv. At the stellar boundary $p_{r}\left(R_{I I}\right)=0$,

$\mathrm{v}$. The metric functions of the core region should match smoothly with the metric functions of the envelope region, and

vi. The metric functions of the envelope region should match smoothly to the Schwarzschild exterior metric.

\section{The Interior}

We use the neutral subcase of the exact solution of [19] with a quadratic equation of state for the core region. Incorporating a nonlinear term in the equation of state leads to a consistent model and generates expressions for the mass, radius and central density consistent with observed stellar 
objects. This quadratic choice of the equation of state ensures that the pressure is higher in the core region. This is the motivation for choosing the results of [19] for region I. In the range $0 \leq r \leq R_{I}$ we have the potentials

$$
\begin{gathered}
e^{2 v_{I}}=\frac{1+a r^{2}}{1+b r^{2}}, \\
e^{2 v_{I}}=B\left(1+a r^{2}\right)^{2 m_{I}}\left(1+b r^{2}\right)^{2 n_{I}} \exp \left[2 F_{I}(r)\right],
\end{gathered}
$$

where

$$
\begin{gathered}
F_{I}(r)=\gamma\left[\frac{2(2 b-a)\left(1+a r^{2}\right)+(b-a)}{2\left(1+a r^{2}\right)^{2}}\right], \\
m_{I}=\gamma[2(a-b)]^{2}\left[\frac{b^{2}}{(b-a)^{3}}+\frac{b}{(b-a)^{2}}+\frac{1}{4}\right], \\
n_{I}=\frac{(a-b)}{4 b}+\gamma[2(a-b)]^{2} \times\left[\frac{b^{2}}{(b-a)^{3}}+\frac{b}{(b-a)^{2}}+\frac{1}{4}\right] .
\end{gathered}
$$

The parameter $\gamma$ arises in the exact models of [19] and we have retained the same parameter for consistency. The various parameters will be constrained when we perform the matching across the different regions is Sec. 4. Then the matter variables are given by

$$
\begin{gathered}
\rho=\frac{(a-b)\left(3+a r^{2}\right)}{\left(1+a r^{2}\right)^{2}}, \\
p_{r}=\gamma \rho^{2},
\end{gathered}
$$

This form of the equation of state produces core densities consistent with earlier treatments as shown by [22]. We note that the gravitational potentials and matter variables are regular and well defined in the core.

We utilize the neutral subcase of the exact models [21] with a linear equation of state for the envelope. This class of solutions is helpful in describing non-homogeneous stars, quark stars and distributions with strange matter. [23] in a comprehensive treatment showed that these models actually generate mass and radius values consistent with recent updated estimates for relativistic compact objects. This is our motivation for selecting the results of [21] for region $I I$. The potentials in the range $R_{I} \leq r \leq R_{I I}$ are

$$
\begin{aligned}
& e^{2 v_{I I}}=\frac{1+a r^{2}}{1+b r^{2}}, \\
& e^{2 v_{I I}}=D\left(1+a r^{2}\right)^{2 m_{I I}}\left(1+b r^{2}\right)^{2 n_{I I}} \exp \left[2 F_{I}(r)\right],
\end{aligned}
$$

where

$$
\begin{gathered}
F_{I I}(r)=\frac{a \beta r^{2}}{4 b}, \\
b=-\frac{a^{3} \alpha R_{I}^{6}+5 a^{2} \alpha R_{I}^{4}}{2 \gamma\left(a^{2} R_{I}^{4}+6 a R_{I}^{2}+9\right)}-\frac{\left(a R_{I}^{2}+1\right)^{2}\left(a R_{I}^{2}+3\right) \sqrt{\alpha^{2}-4 \beta \gamma}}{2 \gamma\left(a^{2} R_{I}^{4}+6 a R_{I}^{2}+9\right)}-\frac{7 a \alpha R_{I}^{2}+3 \alpha}{2 \gamma\left(a^{2} R_{I}^{4}+6 a R_{I}^{2}+9\right)}+a \\
D=\frac{\left(R_{I I}-2 M\right)\left(1+a R_{I I}^{2}\right)^{-\alpha / 2} e^{-\frac{\alpha \beta R_{I}^{2}}{4 b c}}}{R_{I I}} \times\left(1+b R_{I I}^{2}\right)^{-\frac{\beta(b-a)}{4 b^{2} c}-\frac{\alpha(\alpha+1)}{4 b}+\frac{3 \alpha}{4}+\frac{1}{4}} \\
B=\frac{D \exp \left(-\frac{\gamma\left(a\left(4 b R_{I}-2 a R_{I}-3\right)+5 b\right.}{2\left(a R_{I}+1\right)^{2}}-\frac{a \beta R_{I}^{2}}{2 b}\right)}{\sqrt[4]{a R_{I}^{2}-R_{I}^{2}(a-b)+1}} \times\left(1+b R_{I}^{2}\right)^{\frac{\gamma(a-3 b)^{2}}{4(a-b)}+\frac{\alpha(\alpha+1)}{2 b}-\frac{\beta(a-b)}{2 b^{2}}-\frac{a}{4 b}+\frac{3 \alpha}{2}} \times\left(1+a R_{I}^{2}\right)^{\alpha-\frac{\gamma(a-3 b)^{2}}{4(a-b)}}
\end{gathered}
$$

$$
\begin{gathered}
m_{I I}=\frac{\alpha}{2}, \\
n_{I I}=\frac{\alpha(a+b)}{4 b}+\frac{(a-b)(b+\beta)}{4 b^{2}} .
\end{gathered}
$$

The parameter $\alpha$ and $\beta$ arise in the solution of [21] and we retain the same parameters for consistency. These parameters will be constrained later in Sec. 4 when we perform the matching. The matter variables are

$$
\begin{array}{r}
\rho=\frac{(a-b)\left(3+a r^{2}\right)}{\left(1+a r^{2}\right)^{2}}, \\
p_{r}=\alpha \rho-\beta,
\end{array}
$$

The gravitational potentials and matter variables are continuous and well behaved in the envelope.

\section{Matching Conditions}

The line elements (6) and (7) must match smoothly at $r=R_{I}$. This produces the conditions

$$
e^{2 \lambda_{I}\left(R_{I}\right)}=e^{2 \lambda_{I I}\left(R_{I}\right)}, e^{2 v_{I}\left(R_{I}\right)}=e^{2 v_{I I}\left(R_{I}\right)}
$$

The line elements (7) and (8) should match smoothly at $r=R_{I I}$. This produces the conditions

$$
e^{2 \lambda_{I I}\left(R_{I I}\right)}=\left(1-\frac{2 M}{R_{I I}}\right)^{-1}, e^{2 v_{I I}\left(R_{I I}\right)}=1-\frac{2 M}{R_{I I}}
$$

The radial pressure should be continuous at $r=R_{I}$

$$
\gamma \rho^{2}\left(R_{I}\right)=\alpha \rho\left(R_{I}\right)-\beta,
$$

is satisfied. The radial pressure must vanish at the boundary of $\operatorname{star} r=R_{I I}$ which gives

$$
\alpha \rho\left(R_{I I}\right)-\beta=0,
$$

The physical relevant quantities are the mass $M$ and the radius $R_{I I}$ of the star. From system (24)-(26), we obtain

$$
M=\frac{(a-b) R_{I I}^{3}}{2\left(1+a R_{I I}^{2}\right)}
$$

which is the mass of composite. In addition the quantity

$$
R_{I I}=\sqrt{\frac{\sqrt{\alpha} \sqrt{a-b} \sqrt{\alpha(a-b)+8 \beta}}{2 \alpha \beta}-\frac{1}{\alpha}+\frac{\alpha(a-b)}{2 \alpha \beta}},
$$

defines the radius of the star. Then the constants $b, D$ and $B$ can 
In (27)-(31) the constants a, $\alpha, \beta, \gamma$, and $R_{I}$ are free parameters.

\section{Stellar Objects}

We can show that the core envelope model generated in this paper is consistent with observed astronomical objects. We achieve this by generating masses for particular compact stars. We introduce the following transformations

$$
\widetilde{a}=a \mathcal{T}^{2}, \widetilde{b}=b \mathcal{T}^{2},
$$

where $\mathcal{T}$ is a parameter with dimension of length, which helps in comparing with the results of earlier investigations. With the choice of $\widetilde{a}=1, \beta=0.00162$ and the values of $\widetilde{b}, \alpha$ and $\gamma$ given in Table 1 , we can generate specific numerical quantities for the core radius $R_{I}$, the envelope radius $R_{I I}$ and the stellar mass $M$ for the objects PSR J16142230, Vela X-1, PSR J1903+0327, Cen X-3 and SMC X-1. We find the values for the stellar radius $R_{I I}$ is in the range $9.13-10.30 \mathrm{~km}$ and the mass is the range $1.29-1.97 M_{\odot}$.

We note the consistency of these values with the treatment of [20] who considered exact solutions to the Einstein equations with equation of state in the absence of charge. We note that similar values for the mass were obtained by $[20$, 23. 25]. We have also given the mass-radius $\frac{M}{R_{I}}$ relationship for the five compact objects in Table 1. We find that the [26] limit of $\frac{2 M}{R_{I I}}<\frac{8}{9}$ is satisfied.

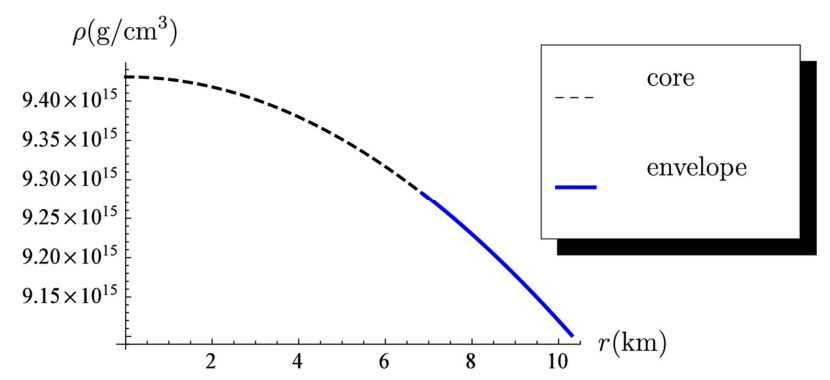

Figure 1. Energy density $\rho$ versus radius $r$ for PSR J1614-2230 $\widetilde{a}=1$, $\widetilde{b}=-25.7351, \alpha=0.1029, R_{I}=6.87 \mathrm{~km}, \gamma=0.1245, \beta=0.00162$, $\left.R_{I I}=10.30 \mathrm{~km}, M=1.97 M_{\odot}\right)$.

$$
p_{r}\left(\text { dyne } / \mathrm{cm}^{2}\right)
$$

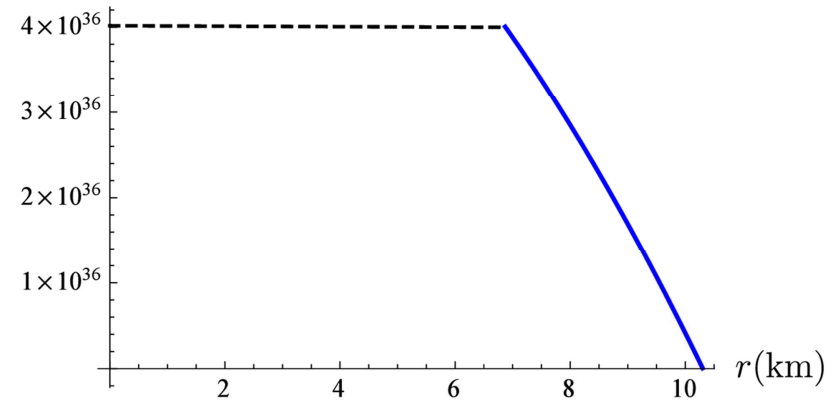

Figure 2. Radial pressure $p_{r}$ versus radius $r$ for PSR J1614-2230 ( $\widetilde{a}=1$, $\widetilde{b}=-25.7351, \alpha=0.10296, R_{I}=6.87 \mathrm{~km}, \gamma=0.1245, \beta=0.00162$, $\left.R_{I I}=10.30 \mathrm{~km}, M=1.97 M_{\odot}\right)$.

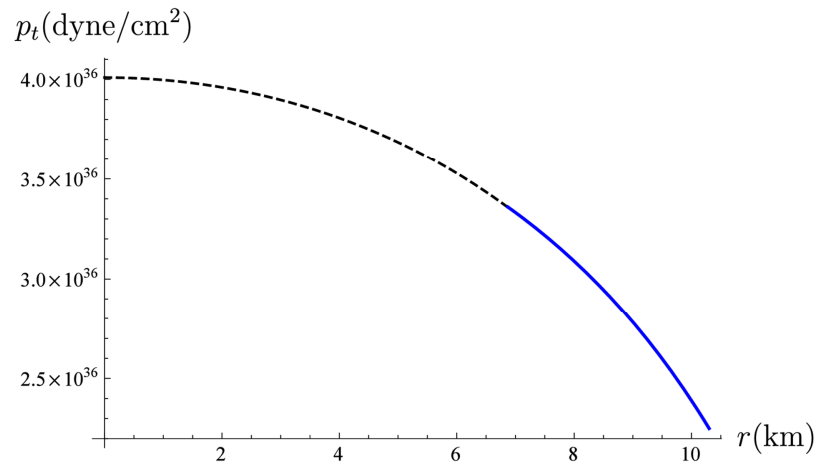

Figure 3. Tangential pressure $p_{t}$ versus radius $r$ for PSR J1614-2230 $\left(\widetilde{a}=1, \widetilde{b}=-25.7351, \alpha=0.10296, R_{I}=6.87 \mathrm{~km}, \gamma=0.1245\right.$, $\left.\beta=0.00162, R_{I I}=10.30 \mathrm{~km}, M=1.97 M_{\odot}\right)$.

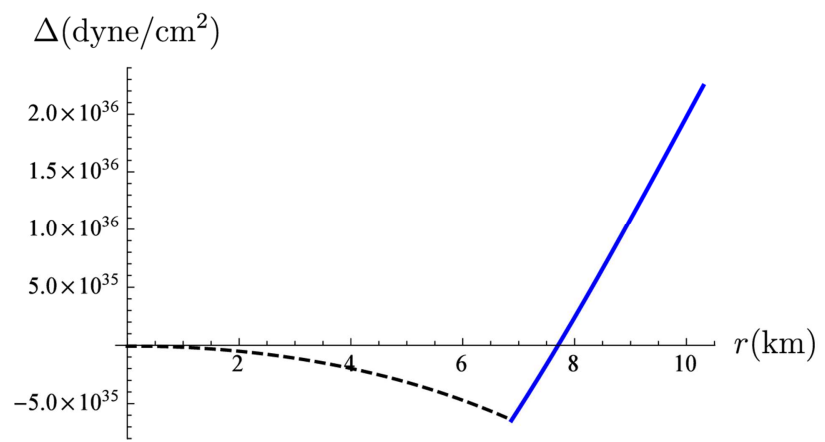

Figure 4. Anisotropy $\Delta$ versus radius $r$ for PSR J1614-2230 ( $\widetilde{a}=1$, $\widetilde{b}=-25.7351, \alpha=0.10296, R_{I}=6.87 \mathrm{~km}, \gamma=0.1245, \beta=0.00162$, $\left.R_{I I}=10.30 \mathrm{~km}, M=1.97 M_{\odot}\right)$.

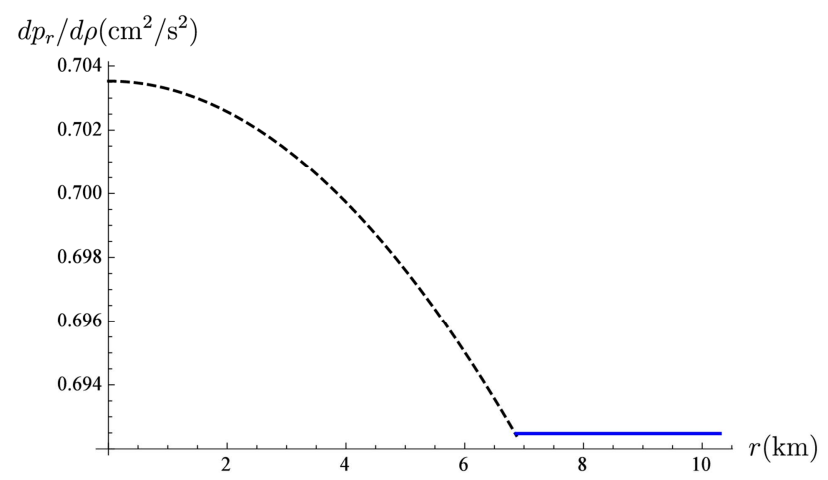

Figure 5. Speed of sound $\frac{d p_{r}}{d \rho}$ versus radius $r$ for PSR J1614-2230 $(\widetilde{a}=1$, $\widetilde{b}=-25.7351, \alpha=0.10296, R_{I}=6.87 \mathrm{~km}, \gamma=0.1245, \beta=0.00162$, $\left.R_{I I}=10.30 \mathrm{~km}, M=1.97 M_{\odot}\right)$.

\section{The Object PSR J1614-2230}

The astronomical object PSR J1614-2230 has been studied by [25]. It has the feature that the recent accurate measurement of its mass gives one of the strongest constraints on the equation of state of superdense matter. The compactification factor for PSR J1614-2230 is $\frac{M}{R_{I I}}=0.191$. This value lies in the range for neutron stars and ultracompact stars. Similar values of the compactification factor have been obtained by [20] when analyzing the field equations for anisotropic compact stars with a linear equation of state, and [23] when studying the field equations by 
incorporating the effect of a nonlinear term in the equation of state. We now perform a detailed physical analysis of the pulsar PSR J164-2230 with a core envelope description.

In order to have detailed analysis of various physical conditions throughout the star we have considered a particular star PSR J1614-2230 having mass $1.97 M_{\odot}$. We choose the radius of the envelope to be $R_{I I}=10.30 \mathrm{~km}$. The same radius value was found analytically in previous investigations by [20] when analyzing the field equations for a non-homogeneous stellar body with a linear equation of state and [23] when studying the non-homogeneous field equations with a quadratic equation of state. Recently [27] by using the [20] model; have studied the stability of the object PSR J1614-2230 by using the same radius and mass values mentioned above. We have assumed the core radius to be the two-thirds of the envelope $R_{I}=\frac{2}{3} R_{I I}=6.87 \mathrm{~km}$. The variation of $d p_{r} / d \rho$ represented in Table 1 for different choices of parameter $b$, can be interpreted as response of geometry to a variation of equation of state for given values of mass and radius of a star. The values of the core radius $R_{I}$ and the envelope radius $R_{I I}$ depend on the choice of parameters in the model. We fix the stellar mass to be 1.97 $M_{\odot}$ and the radius of the envelope to be $R_{I I}=10.30 \mathrm{~km}$ in Table 2. We find that the core radius changes and the envelope radius is fixed. Therefore it is possible for the core to become smaller and more compact. In Table 3 the mass stellar is $1.97 M_{\odot}$ and the radius of the core is taken to be $R_{I}$ $=6.87 \mathrm{~km}$. In order to keep the value of the mass $1.97 M_{\odot}$ constant, we allow the parameters $\alpha$ and $\gamma$ to vary and fix the core radius $R_{I}$. We note that as $\alpha$ increases, the envelope radius $R_{I I}$ increases as well, however the parameter $\widetilde{b}$ which is related to the central density decreases. We find the envelope radius changes and the core radius is fixed. Thus the envelope becomes larger and less compact.
The gravitational potentials $e^{2 \lambda}$ and $e^{2 v}$ are well behaved throughout the star with smooth matching between the core, envelope and the Schwarshild exterior. Figures 1-5 are schematic representations of the energy density $\rho$ the radial pressure $p_{r}$, the tangential pressure $p_{t}$, the measure of anisotropy $\Delta$ and the speed of sound $\frac{d p_{r}}{d \rho}$. These figures have been generated for the parameter values $\widetilde{a}=1, \alpha=$ $0.1029, R_{I}=6.87 \mathrm{~km}, \gamma=0.1245$ and $\beta=0.00162$.

In figures 1-5 the dotted line represents the core and the solid line represents the envelope. In all figures there is smooth matching at the interface between the core and the envelope when $R_{I}=R_{I I}$. The matter variables are all finite at the centre. In Figure 1, we observe that the energy density $\rho$ is a decreasing function so that $\rho^{\prime}<0$ throughout the star. The radial pressure $p_{r}$ is also a decreasing function in Figure 2 ; the pressure $p_{r}$ decreases slightly in the core and more rapidly in the envelope. The rectangular box inside Figure 2 shows the zoom profile for $p_{r}$ in the core which is decreasing. The radial pressure vanishes at the boundary so that $p_{r}(10.30)=0$. In Figure 3 , the tangential pressure $p_{t}$ is also a decreasing function. This behavior for $p_{t}$ is also evident in the works of [24]. The profile for the anisotropy $\Delta$ is given in Figure 4 . We observe that $\Delta$ is initially decreasing in the core and becomes an increasing function in the envelope. The anisotropy $\Delta$ vanishes at the center which is necessary for stability. The profile for the speed of sound $\frac{d p_{r}}{d \rho}$ is given in Figure 5.

The gradient is largest in the core and least in the envelope. However $\frac{d p_{r}}{d \rho}<1$ throughout the star and the speed of light is greater than the speed of sound. Therefore the matter variables are regular and well behaved in both the core and the envelope regions.

Table 1. Mass-radius relationship of some pulsar stars.

\begin{tabular}{llllllll}
\hline$\widetilde{b}$ & $\boldsymbol{\alpha}$ & $\boldsymbol{\gamma}$ & $\boldsymbol{R}_{\boldsymbol{I}}(\mathbf{k m})$ & $\boldsymbol{R}_{\boldsymbol{I I}}(\mathbf{k m})$ & $\boldsymbol{M}_{\boldsymbol{I}}\left(\boldsymbol{M}_{\odot}\right)$ & $\frac{\boldsymbol{M}}{\boldsymbol{R}_{\boldsymbol{I}}}$ & $\mathbf{S T A R}$ \\
\hline-25.7351 & 0.1029 & 0.1245 & 6.87 & 10.30 & 1.97 & 0.191 & PSR J1614-2230 \\
-25.2941 & 0.1238 & 0.1237 & 6.56 & 9.99 & 1.77 & 0.177 \\
-25.055 & 0.105 & 0.1236 & 6.39 & 9.82 & 1.667 & 0.170 & Vela X-1 \\
-24.6104 & 0.1068 & 0.1226 & 6.08 & 9.51 & 1.49 & 0.157 & PSR J1903-327 \\
-24.023 & 0.109 & 0.1219 & 5.70 & 9.13 & 1.29 & 0.141 & Cen X-3 \\
\hline
\end{tabular}

Table 2. Changing core radius values for PRS J1614-2230 structures.

\begin{tabular}{llllll}
\hline$\widetilde{\boldsymbol{b}}$ & $\boldsymbol{\alpha}$ & $\boldsymbol{\gamma}$ & $\boldsymbol{R}_{\boldsymbol{I}}(\mathbf{k m})$ & $\boldsymbol{R}_{\boldsymbol{I I}}(\mathbf{k m})$ & $\boldsymbol{M}_{\boldsymbol{I}}\left(\boldsymbol{M}_{\odot}\right)$ \\
\hline-25.7351 & 0.1029 & 0.1245 & 6.87 & 10.30 & 1.97 \\
-25.7351 & 0.1029 & 0.1381 & 6.37 & 10.30 & 1.97 \\
-25.7351 & 0.1029 & 0.1490 & 5.94 & 10.30 & 1.97 \\
-25.7351 & 0.1029 & 0.1583 & 5.54 & 10.30 & 1.97 \\
-25.7351 & 0.1029 & 0.1670 & 5.14 & 10.30 & 1.97 \\
\hline
\end{tabular}

Table 3. Changing envelope radius values for PRS J1614-2230 structures.

\begin{tabular}{llllll}
\hline$\widetilde{\boldsymbol{b}}$ & $\boldsymbol{\alpha}$ & $\boldsymbol{\gamma}$ & $\boldsymbol{R}_{\boldsymbol{I}}(\mathbf{k m})$ & $\boldsymbol{R}_{\boldsymbol{I I}}(\mathbf{k m})$ & $\boldsymbol{M}_{\boldsymbol{I}}\left(\boldsymbol{M}_{\odot}\right)$ \\
\hline-25.7351 & 0.1029 & 0.1245 & 6.87 & 10.30 & 1.97 \\
-22.2398 & 0.1188 & 0.1943 & 6.87 & 10.30 & 1.97 \\
-19.3339 & 0.1362 & 0.2944 & 6.87 & 10.30 & 1.97 \\
-16.8980 & 0.1554 & 0.4348 & 6.87 & 10.30 & 1.97 \\
-14.8405 & 0.1762 & 0.6281 & 6.87 & 10.30 & 1.97 \\
\hline
\end{tabular}

\section{Conclusion}

In this work we used the core envelope description in order to study an uncharged non-homogeneous star. We chose two different equations of state for the two inner regions. The quadratic equation of state in the core and the linear equation of state in the envelope were utilized. The pressure in the core is higher than the pressure in the envelope. We showed explicitly that the core, envelope and the exterior match smoothly at their interfaces. The masses, radii and compactification factors of five stellar compact objects PSR J1614-2230, PSR J1903+0327, Vela X-1, SMC X-1, Cen X-3 were found. These results are consistent with the works of [20] and [23]. We plotted some physical quantities related to 
the compact star PSR J1614-2230. The matter variables and potentials are well behaved throughout the star, and match smoothly between the two inner regions. We showed that values for the radius of the core and the radius of the envelope can vary by selecting different parameters values. This enables us to consider hybrid models with varying compactness in the core and the envelope. In future work it would be interesting to investigate the effect of different equations of state and other forms of energy momentum tensor on the core envelope model. Finally we remark that in our approach we have considered the macroscopic features of a general relativistic gravitating object; a general description will require an analysis of the microscopic physics which would assist in constraining the equation of state of nuclear matter.

\section{References}

[1] E. Witten, Phys. Rev. D 30 272, (1984).

[2] E. Farhi and R. L. Jaffe, Phys. Rev. D 30 2379, (1984).

[3] R. Sharma and S. Mukherjee, Mod. Lett. A 17, 2535, (2002).

[4] B. C. Paul and R. Tikekar, Gravit. Cosmol. 11, 244, (2005).

[5] R. Tikekar and K. Jotania, Gravit. Cosmol. 15, 129, (2009).

[6] M. C. Durgapal and G. L. Gehlot, Phys. Rev. D 1830, 1102, (1969).

[7] M. C. Durgapal and G. L. Gehlot, J. Phys. Rev. A 4, 749, (1971).

[8] R. S. Fuloria, M. C. Durgapal and S. C. Pande, Astrophys. Space Sci. 148, 95, (1988).

[9] R. S. Fuloria, M. C. Durgapal and S. C. Pande, Astrophys. Space Sci. 151, 255, (1989).

[10] P. S. Negi, A. K. Pande and M. C. Durgapal, Gen. Relativ. Gravit. 22, 735, (1989).
[11] P. S. Negi, A. K. Pande and M. C. Durgapal, Astrophys. Space Sci. 167, 41, (1990).

[12] R. Sharma and S. Mukherjee, Mod. Phys. Lett. A 16, 1049, (2001).

[13] R. Tikekar and V. O. Thomas, Pramana - J. Phys. 64, 5, (2005).

[14] V. O. Thomas, B. S. RAtanpal and P. C. Vinodkumar, Int. - J. Mod. Phys. D 14, 85, (2005).

[15] R. Ruderman, Astron. Astrophys. 10, 427, (1972).

[16] R. Sharma and B. S. Ratanpal, Int. J. Mod. Phys. D 13 , 1350074, (2013).

[17] L. Herrera and W. Barreto, Phys. Rev. D 88, 084022, (2013).

[18] T. Feroze and A. A. Siddiqui, Gen. Relativ. Gravit. 43, 1025, (2011).

[19] S. D. Maharaj and P. Mafa Takisa, Gen. Relativ. Gravit. 44, $1419,(2012)$.

[20] P. Mafa Takisa, S. D. Maharaj and S. Ray, Astrophys. Space Sci. 354, 463, (2014).

[21] P. Mafa Takisa and S. D. Maharaj, Astrophys. Space Sci. 343, $569,(2013)$

[22] S. Thirukkanesh and F. C. Ragel, Pramana - J. Phys. 81, 275 (2013).

[23] P. Mafa Takisa, S. Ray and S. D. Maharaj, Astrophys. Space Sci. 350, 733, (2014).

[24] R. Sharma and S. D. Maharaj, Mon. Not. R. Astron. Soc. 375, 1265, (2007).

[25] T. Gangopadhyay, S. Ray, X-D. Li, J. Dey and M. Dey, Mon. Not. R. Astron. Soc. 431, 3216, (2013).

[26] H. A. Buchdahl, Phys. Rev. 116, 1027, (1959).

[27] M. Azam, S. A. Mardam and M. A. Rehman, Astrophys. Space Sci. 359, 14, (2015). 